\title{
Static Deformation Due to a Long Tensile Fault of Finite Width in an Isotropic Half-Space Welded with an Orthotropic Half-Space
}

\author{
Yogita Godara $^{1^{*}}$, Ravinder Kumar Sahrawat ${ }^{1}$, Mahabir Singh ${ }^{2}$ \\ Deenbandhu Chhotu Ram University of Science and Technology, Murthal, Sonepat-131039. \\ ${ }^{2}$ COE, Deenbandhu Chhotu Ram University of Science and Technology, Murthal, Sonepat-131039, \\ Haryana, (India).
}

Keywords: Isotropic half-space; tensile fault; orthotropic half-space; static-deformation.

\begin{abstract}
Closed-form analytical expressions for displacements and stresses at any point of a two- phase medium consisting of a homogeneous, isotropic, perfectly elastic half-space in welded contact with a homogeneous, orthotropic, perfectly elastic half-space caused by a tensile fault of finite width located at an arbitrary distance from the interface in the isotropic half-space are obtained. The Airy stress function approach is used to obtain the expressions for the stresses and displacements. The vertical tensile fault is considered graphically. The variations of the displacements with the distance from the fault and with depth for various cases have been studied graphically. Also horizontal and vertical displacement of the surface are presented graphically.
\end{abstract}

\section{INTRODUCTION}

Dislocation theory is very useful to determine the static changes that accompany faulting within the earth, which has been discussed by Steketee (1958a, 1958b)[1,2]. As a mathematical model of a fault he uses a displacement dislocation surface, i.e., a surface across which there is a discontinuity in the displacement vector. Using results of Steketee, M.A. Chinnery (1961) [3]deduce the results for deformation of ground around surface faults. The calculation of the deformation caused by shear and tensile faults is necessary for the investigation of seismic and volcanic sources. The solution of the two-dimensional problem of a long inclined shear fault in two welded half-spaces is well known. Tensile fault representation has several very important geophysical applications, such as modelling of the deformation field due to a dyke injection in the volcanic region, mine collapse and fluid driven crack. Recent studies have shown that a large number of earthquake sources cannot be represented by the double-couple source mechanism which models a shear fault. According to Sipkin (1986)[4], the non double-couple mechanism might be due to tensile failure under high fluid pressure.

Maruyama (1964)[5] calculated the Green's functions for two-dimensional elastic dislocations in a semi - infinite medium and obtained surface displacements due to vertical and horizontal rectangular tensile faults in a semi-infinite Poisson solid. Freund and Barnett (1976)[6] developed a 2-D model of dip-slip faulting in a uniform half-space, using the theory of analytic functions of a complex variable and obtained the relationship between fault slip and surface deformation. Davis (1983)[7] modeled the crustal deformation associated with hydro fracture by a dipping rectangular tensile fault beneath the surface of an elastic half-space.

Singh and Garg (1986)[8] obtained the integral expressions for the Airy stress function in an unbounded medium due to various two- dimensional seismic sources. Beginning with these expressions, Rani et al. (1991)[9] obtained the integral expressions for the Airy stress function, displacements and stresses in a homogeneous, isotropic, perfectly elastic half-space due to various two-dimensional sources by applying the traction-free boundary conditions at the surface of the half-space. The integrals were then evaluated analytically, obtaining closed-form expressions for the Airy stress function, the displacements and the stresses at any point of the half-space caused by two-dimensional buried sources. Wu and Chou (1982)[10] applied the generalized method of images to obtain the elastic field of an in-plane line force acting in a two phase orthotropic medium. Singh (1986)[11], and Pan (1989a)[12] studied the static deformation of a transversely isotropic 
multilayered half-space by surface loads. The problem of the static deformation of a transversely isotropic multilayered half-space by buried sources has been discussed by Pan (1989b)[13]. Static deformation of an orthotropic multilayered elastic half-space by twodimensional surface loads has been investigated by Garg et al. (1991)[14].

Singh et al. (1991)[15] followed a similar approach to obtain closed-form analytic expressions for the displacements and the stresses at any point of either of two homogeneous, isotropic, perfectly elastic half- spaces in welded contact due to two-dimensional sources. Kumar et al. (2005)[16] obtained closed-form analytical expressions for the Airy Stress function for a tensile line source in two-welded half-spaces which are integrated analytically to derive the Airy stress function for a tensile fault of finite width.

In the present paper, our aim is to study the two-dimensional deformation of an isotropic half-space in welded contact with an orthotropic half-space due to a long inclined tensile fault of finite width. Beginning with the closed-form expression for the Airy stress function for an arbitrary line source in isotropic half-space in welded contact with an orthotropic half-space given by Singh, J. and Rani, S. (1991)[17] and following Singh and Singh (2000)[18], we obtained Airy stress function for a long tensile fault of arbitrary dip and finite width by analytic integration over the width of the fault. The expressions for the stresses and displacements at any point of the half-space caused by a long vertical tensile fault follow immediately. Two orthotropic materials namely Topaz and Barytes have been considered for numerical computations in case of vertical tensile fault. Numerical results show that the effect of anisotropy on the displacement field is more pronounced when the observer is in the orthotropic half-space.

\section{THEORY}

Let the Cartesian co-ordinates be denoted by $\left(x_{1}, x_{2}, x_{3}\right)$ with $x_{3}$-axis vertically upwards. Consider two homogeneous, perfectly elastic half-spaces which are welded along the plane $\left(x_{1}, x_{2}\right.$, $\left.x_{3}\right)$. The upper half-space $\left(x_{3}>0\right)$ is assumed to be isotropic with stress-strain relation

$$
p_{i j}=2 \mu\left[e_{i j}+\frac{\sigma}{1-2 \sigma} \delta_{i j} e_{k k}\right], \quad(i, j=1,2,3)
$$

where, $p_{i j}$ are the components of stress tensor, $\mathrm{e}_{\mathrm{ij}}$ are the components of strain tensor, $\mu$ is the shear modulus and $\sigma$ is Poisson's ratio. The lower half-space $\left(x_{3}<0\right)$ is assumed to be orthotropic with stress-strain relation.

$$
\left[\begin{array}{l}
p_{11}^{\prime} \\
p_{22}^{\prime} \\
p_{33}^{\prime} \\
p_{23}^{\prime} \\
p_{31}^{\prime} \\
p_{12}^{\prime}
\end{array}\right]=\left[\begin{array}{cccccc}
c_{11} & c_{12} & c_{13} & 0 & 0 & 0 \\
c_{12} & c_{22} & c_{23} & 0 & 0 & 0 \\
c_{13} & c_{23} & c_{33} & 0 & 0 & 0 \\
0 & 0 & 0 & c_{44} & 0 & 0 \\
0 & 0 & 0 & 0 & c_{55} & 0 \\
0 & 0 & 0 & 0 & 0 & c_{66}
\end{array}\right]\left[\begin{array}{c}
e_{11}^{\prime} \\
e_{22}^{\prime} \\
e_{33}^{\prime} \\
2 e_{23}^{\prime} \\
2 e_{31}^{\prime} \\
2 e_{12}^{\prime}
\end{array}\right]
$$

We consider a two dimensional approximation in which displacement component $u_{1}, u_{2}, u_{3}$ are independent of $x_{1}$ so that $\partial / \partial x_{1} \circ 0$. Under this assumption the plane strain problem $\left(u_{1}=0\right)$ and anti-strain problem $\left(u_{2}=0\right.$ and $\left.u_{3}=0\right)$ are decoupled and therefore, can be solved separately. The plane strain problem for an isotropic medium can be solved in terms of Airy stress function $U$ such that

$$
\begin{aligned}
& p_{22}=\partial^{2} U / \partial x_{3}^{2}, p_{33}=\partial^{2} U / \partial x_{2}^{2}, p_{23}=-\partial^{2} U / \partial x_{2} \partial x_{3} \\
& \nabla^{2} \nabla^{2} U=0
\end{aligned}
$$

The plane strain problem for an orthotropic medium can be solved in terms of the Airy Stress function $U^{*}$ such that Garg et al (1991)

$$
p_{22}^{\prime}=\partial 2 U^{*} / \partial x_{3}^{2}, p_{33}^{\prime}=\partial 2 U^{*} / \partial x_{2}^{2}, p_{23}^{\prime}=-\partial 2 U^{*} / \partial x_{2} \partial x_{3},
$$




$$
\begin{aligned}
& \left(a^{2} \frac{\partial^{2}}{\partial x_{2}^{2}}+\frac{\partial^{2}}{\partial x_{3}^{2}}\right)\left(b^{2} \frac{\partial^{2}}{\partial x_{2}^{2}}+\frac{\partial^{2}}{\partial x_{3}^{2}}\right) U^{*}=0 \\
& a^{2}+b^{2}=\frac{\left(c_{22} c_{33}-c_{23}^{2}-2 c_{23} c_{44}\right)}{c_{33} c_{44}}, \quad a^{2} b^{2}=\frac{c_{22}}{c_{33}}
\end{aligned}
$$

For an isotropic medium,

$$
\begin{aligned}
& c_{11}=c_{22}=c_{33}=\frac{2 \mu(1-\sigma)}{\frac{1-2 \sigma}{2 \mu \sigma}} \\
& c_{12}=c_{13}=c_{23}=\frac{2-2 \sigma}{1-2 \sigma} \\
& c_{44}=c_{55}=c_{66}=\mu
\end{aligned}
$$

This yields $a^{2}=b^{2}=1$ and equation (6) reduces to equation (4).

As given by Rani et al. (1991), we have the Airy stress function for an arbitrary line source parallel to the $x_{1}$-axis and passing through the point $\left(y_{2}, y_{3}\right)$ located in the isotropic half-space welded with the orthotropic half- space.

For the isotropic half-space,

$$
\begin{aligned}
U=L_{0} \tan ^{-1}\left(\frac{x_{2}-y_{2}}{\left|x_{3}-y_{3}\right|}\right)+M_{0} \frac{\left(x_{2}-y_{2}\right)\left|x_{3}-y_{3}\right|}{R^{2}}-P_{0} \ln R+Q_{0} \frac{\left(x_{3}-y_{3}\right)^{2}}{R^{2}} \\
+L^{-}\left\{X_{1} \tan ^{-1}\left(\frac{x_{2}-y_{2}}{x_{3}+y_{3}}\right)+\frac{2 X_{2}\left(x_{2}-y_{2}\right) x_{3}}{S^{2}}\right\} \\
+M^{-}\left\{2(D-C) \tan ^{-1}\left(\frac{x_{2}-y_{2}}{x_{3}+y_{3}}\right)+\frac{\left(X_{1} y_{3}+X_{3} x_{3}\right)\left(x_{2}-y_{2}\right)}{S^{2}}+\frac{\left.4 X_{2} y_{3}\left(x_{2}-y_{2}\right) x_{3}\left(x_{3}+y_{3}\right)\right\}}{S^{4}}\right\} \\
+P^{-}\left\{-X_{1} \ln S+\frac{2 X_{2} x_{3}\left(x_{3}+y_{3}\right)}{S^{2}}\right\} \\
+Q^{-}\left\{-2(D-C) \ln S+\frac{\left(X_{1} y_{3}+X_{3} x_{3}\right)\left(x_{3}+y_{3}\right)-2 X_{2} x_{3} y_{3}}{S^{2}}+\frac{4 X_{2} y_{3} x_{3}\left(x_{3}+y_{3}\right)^{2}}{S^{4}}\right\}
\end{aligned}
$$

and for orthotropic half-space,

$$
\begin{aligned}
& U^{*}=2 L^{-}\left\{A \tan ^{-1}\left(\frac{x_{2}-y_{2}}{y_{3}-a x_{3}}\right)+B \tan ^{-1}\left(\frac{x_{2}-y_{2}}{y_{3}-b x_{3}}\right)\right\} \\
&+2 M^{-}\left\{-C \tan ^{-1}\left(\frac{x_{2}-y_{2}}{y_{3}-a x_{3}}\right)+D \tan ^{-1}\left(\frac{x_{2}-y_{2}}{y_{3}-b x_{3}}\right)+\left(x_{2}-y_{2}\right) y_{3}\left(\frac{A}{T^{2}}+\frac{B}{H^{2}}\right)\right\} \\
&- 2 P^{-}\{A \ln T+B \ln H\} \\
&+2 Q^{-}\left\{C \ln T+\frac{A y_{3}\left(y_{3}-a x_{3}\right)}{T^{2}}-D \ln H+\frac{B y_{3}\left(y_{3}-b x_{3}\right)}{H^{2}}\right\}
\end{aligned}
$$

Where, $\left(x_{2}, x_{3}\right)=$ receiver location,

$$
\begin{aligned}
& R^{2}=\left(x_{2}-y_{2}\right)^{2}+\left(x_{3}-y_{3}\right)^{2} \quad S^{2}=\left(x_{2}-y_{2}\right)^{2}+\left(x_{3}+y_{3}\right)^{2} \\
& T^{2}=\left(x_{2}-y_{2}\right)^{2}+\left(y_{3}-a x_{3}\right)^{2} \quad H^{2}=\left(x_{2}-y_{2}\right)^{2}+\left(y_{3}-b x_{3}\right)^{2} \\
& x_{3} \neq y_{3}, \quad x_{2} \neq y_{2}, \quad a x_{3} \neq y_{3}, \quad b x_{3} \neq y_{3}, \\
& X_{1}=2(A+B)-1, \quad X_{2}=A(1+a)+B(1+b)-1, \\
& X_{3}=2 D(1+b)-2 C(1+a)+1 \text {, } \\
& A=\frac{\alpha\left(1-b+2 \mu s_{2}-2 \mu r_{2}\right)}{W}, \quad B=\frac{\alpha\left(a-1+2 \mu r_{1}-2 \mu s_{1}\right)}{W} \\
& C=\frac{\left(1+b-b \alpha+2 \mu \alpha s_{2}\right)}{W}, \quad D=\frac{\left(1+a-a \alpha+2 \mu \alpha s_{1}\right)}{W}, \\
& r_{1}=\frac{c_{33} a^{2}+c_{23}}{\Delta}, \quad r_{2}=\frac{c_{33} b^{2}+c_{23}}{\Delta}, \\
& s_{1}=\frac{c_{23} a^{2}+c_{22}}{a \Delta}, \quad s_{2}=\frac{c_{23} b^{2}+c_{22}}{b \Delta}, \quad \Delta=\left(c_{22} c_{33}-c_{23}^{2}\right) \text {, } \\
& W=\left(1+a-\alpha+2 \mu \alpha r_{1}\right)\left(1+b-b \alpha+2 \mu \alpha s_{2}\right) \\
& -\left(1+a-a \alpha+2 \mu \alpha s_{1}\right)\left(1+b-\alpha+2 \mu \alpha r_{2}\right), \quad L_{0}, M_{0}, P_{0}, Q_{0} \\
& x_{3}<y_{3}
\end{aligned}
$$


And in equations (9), (10), L0, M0, P0, Q0 are the source coefficients and $L^{-}, M^{-}, P^{-}, Q^{-}$are the values of these source coefficients valid for $\mathrm{x}_{3}<\mathrm{y}_{3}$.Singh and Garg (1986) and Singh and Rani (1991) have given these source coefficients for various seismic sources. For ready reference, the relevant source coefficients are given in Appendix. Let $U_{i}$ denote the Airy stress function at an arbitrary point $\mathrm{P}\left(x_{2}, x_{3}\right)$ for a unit concentrated force acting at the point $\mathrm{Q}\left(y_{2}, y_{3}\right)$ in the $x_{i}$ direction.Then, the Airy stress function for a long fault can be expressed as a line integral (Maruyama, 1964).

$$
U=\int_{0}^{2} \Delta u_{i} U_{i j} n_{j} d s
$$

wnere, tne summation convention has been used (the suffices can assume the values 2 and 3 only). In equation (12), $\Delta u_{i}$ is the displacement dislocation vector; $n_{j}$ is the unit normal to the fault; and

$$
U_{i j}=U_{j i}=\lambda \delta_{i j} \frac{\partial}{\partial y_{k}} U_{k}+\mu\left(\frac{\partial}{\partial y_{i}} U_{j}+\frac{\partial}{\partial y_{j}} U_{i}\right)
$$

For a tensile fault, vector $\Delta u_{i}$ is parallel to the normal $n_{i}$ to the fault. Therefore, if $b$ ' is the magnitude of $a \Delta u_{i}$ is $\delta$ re dip angle (figure 1(a)), we have

$$
\begin{aligned}
& \Delta u_{2}=-b^{\prime} \sin \delta, \quad \Delta u_{3}=b^{\prime} \cos \delta \\
& n_{2}=-\sin \delta, \quad n_{3}=\cos \delta,
\end{aligned}
$$

Using equations (13) and (14) in equation (12), we get the following expression for the Airy stress function for a line source .

$$
U=b^{\prime} d s\left[U_{22} \sin ^{2} \delta-U_{23} \sin 2 \delta+U_{33} \cos ^{2} \delta\right]
$$

$d s$ is the width of the line fault. Therefore, the Airy stress function for a long tensile fault of arbitrary dip can be expressed as a linear combination of

i) $b^{\prime} d s U_{22}$, the Airy stress function for a vertical tensile fault $\left(\delta=90^{\circ}\right)$ with dislocation in the $x_{2-}$ direction;

ii) $b^{\prime} d s U_{33}$, the Airy stress function for a horizontal tensile fault $\left(\delta=0^{\circ}\right)$ with dislocation in the $x_{3-\text { direction; }}$

iii) $b^{\prime} d s U_{23}$, the Airy stress function for a vertical dip-slip fault.

Using the values of the source coefficients $L_{0}, M_{0}, P_{0}, Q_{0}, L^{-}, M^{-}, P^{-}, Q^{-}$given in Appendix, equations (9), (10), (15) yield the Airy stress function due to a long tensile line source of

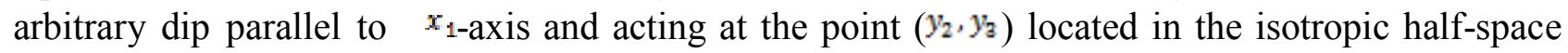
welded with the orthotropic half-space in the form

For isotropic half-space

$$
\begin{aligned}
U=\frac{\alpha \mu b^{\prime} d s}{\pi}[- & \ln R-X_{1} \ln S+\frac{2 X_{2} x_{3}\left(x_{3}+y_{3}\right)}{S^{2}}+\cos 2 \delta\left\{\frac{\left(x_{3}-y_{3}\right)^{2}}{R^{2}}\right. \\
& \left.-2(D-C) \ln S+\frac{\left(X_{1} y_{3}+X_{3} x_{3}\right)\left(x_{3}+y_{3}\right)-2 X_{2} x_{3} y_{3}}{S^{2}}+\frac{4 X_{2} x_{3} y_{3}\left(x_{3}+y_{3}\right)^{2}}{S^{4}}\right\} \\
& -\sin 2 \delta\left\{\frac{\left(x_{2}-y_{2}\right)\left(x_{3}-y_{3}\right)}{R^{2}}-\frac{\left(X_{1} y_{3}+X_{3} x_{3}\right)\left(x_{2}-y_{2}\right)}{S^{2}}\right. \\
& \left.\left.-2(D-C) \tan ^{-1}\left(\frac{x_{2}-y_{2}}{x_{3}+y_{3}}\right)-\frac{4 X_{2} x_{3} y_{3}\left(x_{2}-y_{2}\right)\left(x_{3}+y_{3}\right)}{S^{4}}\right\}\right]
\end{aligned}
$$

and for the orthotropic half space

$$
\begin{aligned}
U^{*}=\frac{2 \alpha \mu b^{\prime} d s}{\pi}[ & -(A \ln T+B \ln H)+\cos 2 \delta\left\{C \ln T-D \ln H+\frac{A y_{3}\left(y_{3}-a x_{3}\right)}{T^{2}}+\frac{B y_{3}\left(y_{3}-b x_{3}\right)}{H^{2}}\right\} \\
& \left.-\sin 2 \delta\left\{C \tan ^{-1}\left(\frac{x_{2}-y_{2}}{y_{3}-a x_{3}}\right)-D \tan ^{-1}\left(\frac{x_{2}-y_{2}}{y_{3}-b x_{3}}\right)-\left(x_{2}-y_{2}\right) y_{3}\left(\frac{A}{T^{2}}+\frac{B}{H^{2}}\right)\right\}\right]
\end{aligned}
$$

where, $\quad \alpha=\frac{1}{2(1-\sigma)}$ 
Now, using the polar coordinates $(s, \delta)$, see figure1(a)

$$
\begin{aligned}
& \mathrm{y}_{2}=s \cos \delta, \\
& \$_{21}=\alpha+s \sin \delta
\end{aligned}
$$

anı

$L$ ), we will obtain the following expressions for the Airy stress function for a long tensile fault of width $L$ and infinite length with lower edge of the fault at distance ${ }^{d}$ from the interface:

$$
\begin{aligned}
U=\frac{\alpha \mu b^{\prime}}{\pi}[\{(1+ & \left.\left.X_{1}\right) \cos ^{2} \delta+2(D-C) \cos 2 \delta\right\} s+\left(x_{2} \cos \delta+X \sin \delta-s\right) \ln R \\
& \left.+\left\{2(D-C)\left(x_{2} \cos \delta+X^{\prime} \sin \delta-s \cos 2 \delta\right)+X_{1}\left(x_{2} \cos \delta-d \sin \delta-s\right)-X_{3} x_{3} \sin \delta\right)\right\} \ln S \\
& +2(D-C) s \sin 2 \delta \tan ^{-1}\left(\frac{x_{2}-s \cos \delta}{X^{\prime}+s \sin \delta}\right)-2 X_{2} x_{3}\left(x_{2} \cos \delta-X^{\prime} \sin \delta-s\right)(d+s \sin \delta) \frac{1}{s^{2}} \\
& +\left\{x_{3} \cos \delta\left(-X_{1}+X_{3}+2 X_{2}\right)\right. \\
& \left.\left.-2(D-C)\left(-x_{2} \sin \delta+X^{\prime} \cos \delta\right)\right\} \tan ^{-1}\left(\frac{s-x_{2} \cos \delta+X^{\prime} \sin \delta}{x_{2} \sin \delta+X^{\prime} \cos \delta}\right)\right]\left.\right|_{0} ^{2}
\end{aligned}
$$

$$
\begin{aligned}
U^{*}=\frac{2 \alpha \mu b^{\prime}}{\pi}[\{(D & \left.-C) \cos 2 \delta+(A+B) \cos ^{2} \delta\right\}_{s} \\
& +\left\{A\left(x_{2} \cos \delta-d \sin \delta-s\right)-C\left(x_{2} \cos \delta+Y \sin \delta-s \cos 2 \delta\right)\right\} \ln T \\
& +\left\{B\left(x_{2} \cos \delta-d \sin \delta-s\right)+D\left(x_{2} \cos \delta+Y^{\prime} \sin \delta-s \cos 2 \delta\right)\right\} \ln H \\
& -C s \sin 2 \delta \tan ^{-1}\left(\frac{x_{2}-s \cos \delta}{Y+s \sin \delta}\right) \\
& +D s \sin 2 \delta \tan ^{-1}\left(\frac{x_{2}-s \cos \delta}{Y^{\prime}+s \sin \delta}\right) \\
& +\left\{A a x_{3} \cos \delta-C\left(x_{2} \sin \delta-Y \cos \delta\right)\right\} \tan ^{-1}\left(\frac{s-x_{2} \cos \delta+Y \sin \delta}{x_{2} \sin \delta+Y \cos \delta}\right) \\
& \left.+\left\{B b x_{3} \cos \delta+D\left(x_{2} \sin \delta-Y^{\prime} \cos \delta\right)\right\} \tan ^{-1}\left(\frac{s-x_{2} \cos \delta+Y^{\prime} \sin \delta}{x_{2} \sin \delta+Y^{\prime} \cos \delta}\right)\right]\left.\right|_{0} ^{2}
\end{aligned}
$$

Where now,

$$
\begin{aligned}
& R^{2}=\left(x_{2}-s \cos \delta\right)^{2}+(X-s \sin \delta)^{2}, \quad S^{2}=\left(x_{2}-s \cos \delta\right)^{2}+\left(X^{\prime}+s \sin \delta\right)^{2}, \\
& T^{2}=\left(x_{2}-s \cos \delta\right)^{2}+(Y+s \sin \delta)^{2}, \\
& \left.X=x_{3}-d, \quad X^{\prime}=x_{3}+d, \quad Y=d-a x_{3}, \quad Y_{2}-s \cos \delta\right)^{2}+\left(Y^{\prime}+s \sin \delta\right)^{2},
\end{aligned}
$$

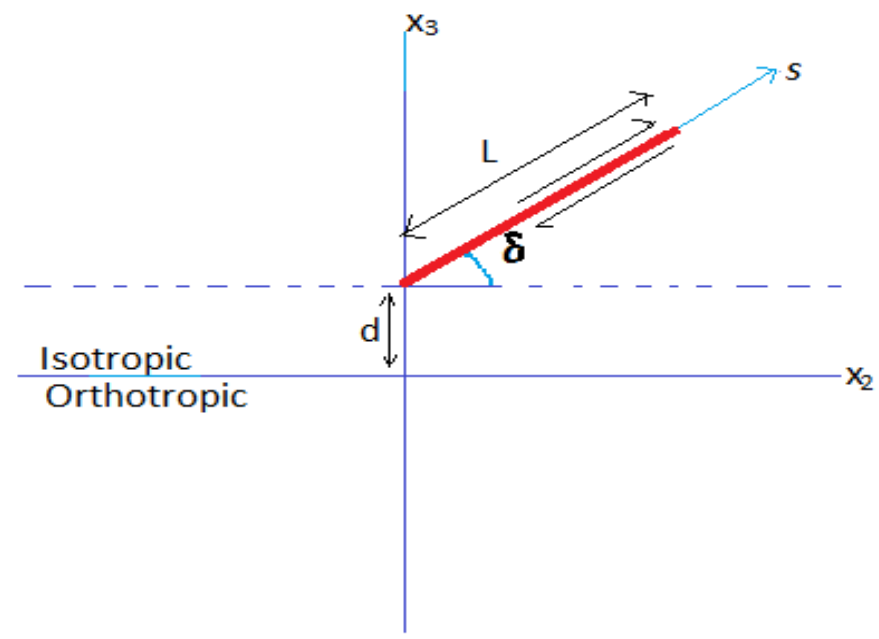

(a) 


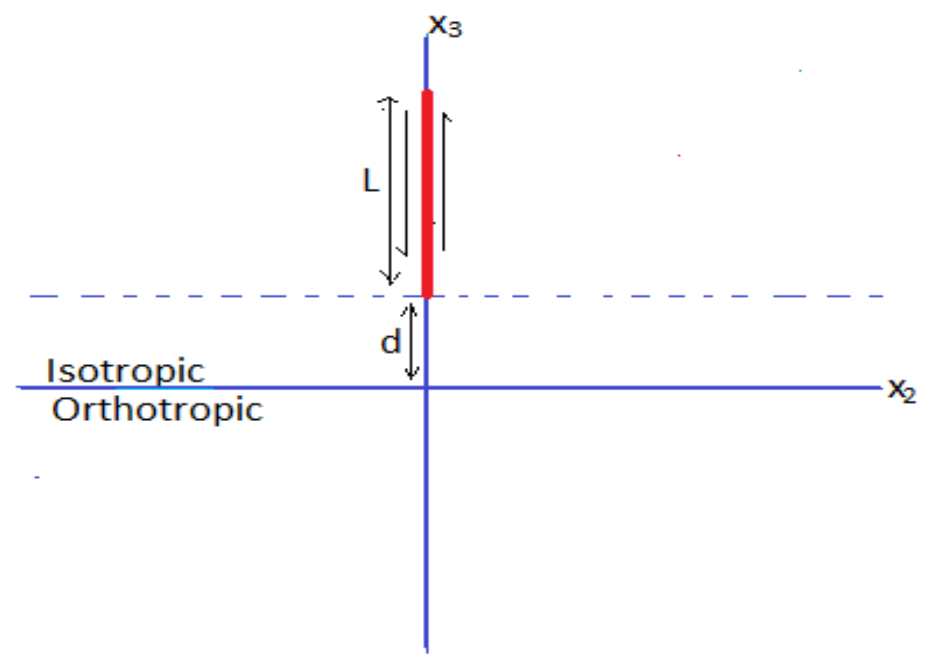

(b)

Figure 1 Geometry of a tensile fault of width L having lower edge at a distance $d$ from the interface in the isotropic half- space in welded contact with an orthotropic half-space (a) $\delta$ is dip angle and $s$ is the distance from the lower edge of the fault, measured in dip direction; (b) $\delta=90^{\circ}$.

\section{STRESSES}

Using equations (3) and (18), we will obtain the following expressions stresses for a long tensile fault of width $L$ and infinite length with lower edge of the fault at distance $d$ from the interface as for isotropic half-space,

$$
\begin{aligned}
p_{22}=\frac{\alpha \mu b^{\prime}}{\pi}\left[\left(x_{2}\right.\right. & \cos \delta+3 X \sin \delta+s \cos 2 \delta) \frac{1}{R^{2}}-2\left(x_{2} \cos \delta+X \sin \delta-s\right)(X-s \sin \delta)^{2} \frac{1}{R^{4}} \\
& +\left\{2\left(X_{3}-D+C\right)\left(x_{2} \cos \delta-X^{\prime} \sin \delta-s\right)+\left(4 X_{2}-X_{1}\right)\left(x_{2} \cos \delta+d \sin \delta-s \cos 2 \delta\right)\right. \\
& \left.-X_{3} x_{2} \sin \delta\right\} \frac{1}{S^{2}} \\
& -2\left\{X_{1}\left(x_{2} \cos \delta-X^{\prime} \sin \delta-s\right)\left(X^{\prime}+s \sin \delta\right)^{2}+2 X_{2} x_{3}^{2} \cos \delta\left(x_{2}-s \cos \delta\right)\right. \\
& -\left(X_{1}-X_{3}\right) x_{3}\left(X^{\prime}+s \sin \delta\right)\left(x_{2} \cos \delta-X^{\prime} \sin \delta-s\right) \\
& \left.-2 X_{2}(d+s \sin \delta)\left(X^{\prime}+s \sin \delta\right)\left[2\left(x_{2} \cos \delta-X^{\prime} \sin \delta-s\right)+3 x_{3} \sin \delta\right]\right\} \frac{1}{S^{4}} \\
& \left.-16 X_{2} x_{3}\left(x_{2} \cos \delta-X^{\prime} \sin \delta-s\right)(d+s \sin \delta)\left(X^{\prime}+s \sin \delta\right)^{2} \frac{1}{S^{6}}\right]\left.\right|_{0} ^{2}
\end{aligned}
$$

$$
\begin{aligned}
p_{23}=\frac{\alpha \mu b^{\prime}}{\pi}[- & \left(x_{2} \sin \delta-X \cos \delta\right) \frac{1}{R^{2}}+2(X-s \sin \delta)^{2}\left(x_{2} \sin \delta-X \cos \delta\right) \frac{1}{R^{4}} \\
& +\left\{-2(D-C)\left(x_{2} \sin \delta+X^{\prime} \cos \delta\right)+\left(-X_{1}+4 X_{2}+X_{3}\right) x_{3} \cos \delta+X_{3}\left(x_{2} \sin \delta+X^{\prime} \cos \delta\right)\right\} \frac{1}{S^{2}} \\
& +\left\{2 x_{3}\left(X_{1}-2 X_{2}\right)\left(X^{\prime}+s \sin \delta\right)^{2} \cos \delta\right) \\
& -2 X_{1}\left(X^{\prime}+s \sin \delta\right)\left[X^{\prime} \cos \delta\left(X^{\prime}+s \sin \delta\right)+d \sin \delta\left(x_{2}-s \cos \delta\right)+s \cos \delta\left(x_{2} \sin \delta+X^{\prime} \cos \delta\right)\right] \\
& +4 X_{2}(d+s \sin \delta)\left[\left(x_{3}+X^{\prime}+s \sin \delta\right)\left(x_{2} \sin \delta+X^{\prime} \cos \delta\right)+2 x_{3} \cos \delta\left(X^{\prime}+s \sin \delta\right)\right] \\
& \left.-2 X_{3} x_{3}\left(X^{\prime}+s \sin \delta\right)\left(x_{2} \sin \delta+X^{\prime} \cos \delta\right)\right\} \frac{1}{S^{4}} \\
& \left.-16 X_{2} x_{3}(d+s \sin \delta)\left(X^{\prime}+s \sin \delta\right)^{2}\left(x_{2} \sin \delta+X^{\prime} \cos \delta\right) \frac{1}{S^{6}}\right]\left.\right|_{0} ^{2}
\end{aligned}
$$




$$
\begin{aligned}
p_{33}=\frac{\alpha \mu b^{\prime}}{\pi}\left[\left(x_{2}\right.\right. & \cos \delta-X \sin \delta-s \cos 2 \delta) \frac{1}{R^{2}} \\
& +2\left(x_{2} \cos \delta+X \sin \delta-s\right)(X-s \sin \delta)^{2} \frac{1}{R^{4}} \\
& +\left\{2(D-C)\left(x_{2} \cos \delta-X^{\prime} \sin \delta-s\right)+X_{1}\left(x_{2} \cos \delta+d \sin \delta-s \cos 2 \delta\right)+X_{3} x_{3} \sin \delta\right\} \frac{1}{S^{2}} \\
& +\left\{2 X_{1}\left(X^{\prime}+s \sin \delta\right)\left[\left(X^{\prime}+s \sin \delta\right)\left(x_{2} \cos \delta-d \sin \delta-s\right)-x_{3} \cos \delta\left(x_{2}-s \cos \delta\right)\right]\right. \\
& +4 X_{2} x_{3}\left[x_{3} \cos \delta\left(x_{2}-s \cos \delta\right)+3 \sin \delta\left(X^{\prime}+s \sin \delta\right)(d+s \sin \delta)\right] \\
& \left.+2 X_{3} x_{3}\left(X^{\prime}+s \sin \delta\right)\left(x_{2} \cos \delta-X^{\prime} \sin \delta-s\right)\right\} \frac{1}{S^{4}} \\
& \left.+16 X_{2} x_{3}\left(x_{2} \cos \delta-X^{\prime} \sin \delta-s\right)(d+s \sin \delta)\left(X^{\prime}+s \sin \delta\right)^{2}\right]\left.\right|_{0} ^{2}
\end{aligned}
$$

Using equations (5) and (19), we will obtain the following expressions stresses for a long tensile fault of width Land infmite length with lower edge of the fault at distance $d$ from the interface as for orthotropic half-space,

$$
\begin{aligned}
& p_{22}^{\prime}=\frac{2 \alpha \mu b^{\prime}}{\pi}\left[a^{2}\left\{A\left(s \cos 2 \delta-x_{2} \cos \delta-d \sin \delta\right)+C\left(x_{2} \cos \delta-Y \sin \delta-s\right)\right\} \frac{1}{T^{2}}\right. \\
& +b^{2}\left\{B\left(s \cos 2 \delta-x_{2} \cos \delta-d \sin \delta\right)-D\left(x_{2} \cos \delta-Y^{\prime} \sin \delta-s\right)\right\} \frac{1}{H^{2}} \\
& -2 a^{2} A(d+s \sin \delta)\left(x_{2} \cos \delta-Y \sin \delta-s\right)(Y+s \sin \delta) \frac{1}{T^{4}} \\
& \left.-2 b^{2} B(d+s \sin \delta)\left(x_{2} \cos \delta-Y^{\prime} \sin \delta-s\right)\left(Y^{\prime}+s \sin \delta\right) \frac{1}{H^{4}}\right]\left.\right|_{0} ^{2} \\
& p_{23}^{\prime}=\frac{2 \alpha \mu b^{\prime}}{\pi}\left[-a\left\{C\left(x_{2} \sin \delta+Y \cos \delta\right)+a A x_{3} \cos \delta\right\} \frac{1}{T^{2}}+b\left\{D\left(x_{2} \sin \delta+Y^{\prime} \cos \delta\right)-b B x_{3} \cos \delta\right\} \frac{1}{H^{2}}\right. \\
& -2 a A(d+s \sin \delta)\left(-x_{2} \sin \delta-Y \cos \delta\right)(Y+s \sin \delta) \frac{1}{T^{4}} \\
& \left.-2 b B(d+s \sin \delta)\left(-x_{2} \sin \delta-Y^{\prime} \cos \delta\right)\left(Y^{\prime}+s \sin \delta\right) \frac{1}{H^{4}}\right]\left.\right|_{0} ^{2} \\
& p_{33}^{\prime}=\frac{2 \alpha \mu b^{\prime}}{\pi}\left[\left\{A\left(x_{2} \cos \delta+d \sin \delta-s \cos 2 \delta\right)-C\left(x_{2} \cos \delta-Y \sin \delta-s\right)\right\} \frac{1}{T^{2}}\right. \\
& +\left\{B\left(x_{2} \cos \delta+d \sin \delta-s \cos 2 \delta\right)+D\left(x_{2} \cos \delta-Y^{\prime} \sin \delta-s\right)\right\} \frac{1}{H^{2}} \\
& +2 A(d+s \sin \delta)\left(x_{2} \cos \delta-Y \sin \delta-s\right)(Y+s \sin \delta) \frac{1}{T^{4}} \\
& \left.+2 B(d+s \sin \delta)\left(x_{2} \cos \delta-Y^{\prime} \sin \delta-s\right)\left(Y^{\prime}+s \sin \delta\right) \frac{1}{H^{4}}\right]\left.\right|_{0} ^{2}
\end{aligned}
$$

\section{DISPLACEMENTS}

The displacements, for the isotropic half-space, are given by the expressions (Singh and Rani (1991))

$$
\begin{aligned}
& 2 \mu u_{2}=-\frac{\partial U}{\partial x_{2}}+\frac{1}{2 \alpha} \int\left(p_{22}+p_{33}\right) d x_{2}, \\
& 2 \mu u_{3}=-\frac{\partial U}{\partial x_{3}}+\frac{1}{2 \alpha} \int\left(p_{22}+p_{33}\right) d x_{3},
\end{aligned}
$$

The displacements, for the orthotropic half-space, are given by the expressions (Singh and Rani (1991)) 


$$
\begin{aligned}
& u_{2}^{\prime}=\frac{1}{\Delta} \int\left(c_{33} p_{22}^{\prime}-c_{23} p_{33}^{\prime}\right) d x_{2}, \\
& u_{3}^{\prime}=\frac{1}{\Delta} \int\left(c_{22} p_{33}^{\prime}-c_{23} p_{22}^{\prime}\right) d x_{3},
\end{aligned}
$$

where, $\Delta=c_{22} c_{33}-c_{23}^{2}$

Using equations (20), (22), (26a), and (26b), we will obtain the following expressions stresses for a long tensile fault of width $L$ and infinite length with lower edge of the fault at didtance from the interface as forisotropic half-space,

$$
\begin{aligned}
& u_{2}=\frac{\alpha b^{\prime}}{2 \pi}\left[\left(\frac{1}{\alpha}-1\right) \cos \delta \ln R-\left\{2(D-C)+X_{1}-\frac{\left(X_{3}+2 X_{2}\right)}{\alpha}\right\} \cos \delta \ln S\right. \\
& +\frac{\sin \delta}{\alpha}\left\{\tan ^{-1}\left(\frac{x_{2}-s \cos \delta}{X-s \sin \delta}\right)-X_{3} \tan ^{-1}\left(\frac{x_{2}-s \cos \delta}{X^{\prime}+s \sin \delta}\right)\right\} \\
& -2(\mathrm{D}-\mathrm{C}) \sin \delta \tan ^{-1}\left(\frac{s-x_{2} \cos \delta+X^{\prime} \sin \delta}{x_{2} \sin \delta+X^{\prime} \cos \delta}\right)-(X-s \sin \delta)\left(x_{2} \sin \delta-X \cos \delta\right) \frac{1}{R^{2}} \\
& +\left\{\left(X_{1}-\frac{2 X_{2}}{\alpha}\right)(d+s \sin \delta)\left(x_{2} \sin \delta+X^{\prime} \cos \delta\right)+x_{3}\left[2 X_{2} x_{3} \cos \delta+X_{3}\left(x_{2} \sin \delta+X^{\prime} \cos \delta\right)\right]\right\} \frac{1}{S^{2}} \\
& \left.+4 X_{2} x_{3}\left(x_{2} \sin \delta+X^{\prime} \cos \delta\right)(d+s \sin \delta)\left(X^{\prime}+s \sin \delta\right) \frac{1}{S^{4}}\right]\left.\right|_{0} ^{2} \\
& u_{3}=\frac{\alpha b^{\prime}}{2 \pi}\left[\left(\frac{1}{\alpha}-1\right) \sin \delta \ln R-\left\{2(D-C)+X_{3}\left(\frac{1}{\alpha}-1\right)\right\} \sin \delta \ln S\right. \\
& +\frac{\cos \delta}{\alpha}\left\{\tan ^{-1}\left(\frac{X-s \sin \delta}{x_{2}-s \cos \delta}\right)+\left(2 X_{2}+X_{3}\right) \tan ^{-1}\left(\frac{X^{\prime}+s \sin \delta}{x_{2}-s \cos \delta}\right)\right\} \\
& +\left\{2(D-C)+X_{1}-2 X_{2}-X_{3}\right\} \cos \delta \tan ^{-1}\left(\frac{s-x_{2} \cos \delta+X^{\prime} \sin \delta}{x_{2} \sin \delta+X^{\prime} \cos \delta}\right) \\
& -(X-s \sin \delta)\left(x_{2} \cos \delta+X \sin \delta-s\right) \frac{1}{R^{2}} \\
& +\left\{\left(-X_{1}+2 X_{2}-\frac{2 X_{2}}{\alpha}\right)(d+s \sin \delta)\left(x_{2} \cos \delta-X^{\prime} \sin \delta-s\right)\right. \\
& \left.+2 X_{2} x_{3}\left(s \cos 2 \delta-x_{2} \cos \delta-d \sin \delta\right)-X_{3} x_{3}\left(\left(x_{2} \cos \delta-X^{\prime} \sin \delta-s\right)\right)\right\} \frac{1}{S^{2}} \\
& \left.-4 X_{2} x_{3}\left(x_{2} \cos \delta-X^{\prime} \sin \delta-s\right)(d+s \sin \delta)\left(X^{\prime}+s \sin \delta\right) \frac{1}{s^{4}}\right]\left.\right|_{0} ^{2}
\end{aligned}
$$

Using equations (23), (25), (27a), and (27b), we will obtain the following expressions stresses for a long tensile fault of width $L$ and infinite length with lower edge of the fault at distance $d$ from the interface as for orthotropic half-space,

$$
\begin{aligned}
& u_{2}^{\prime}=-\frac{2 \alpha \mu b^{\prime}}{\pi}\left[(A-C) r_{1} \cos \delta \ln T+(B+D) r_{2} \cos \delta \ln H\right. \\
& -A r_{1}(d+s \sin \delta)\left(x_{2} \sin \delta+Y \cos \delta\right) \frac{1}{T^{2}}-B r_{2}(d+s \sin \delta)\left(x_{2} \sin \delta+Y^{\prime} \cos \delta\right) \frac{1}{H^{2}} \\
& \left.+C r_{1} \sin \delta \tan ^{-1}\left(\frac{x_{2}-s \cos \delta}{Y+s \sin \delta}\right)-D r_{2} \sin \delta \tan ^{-1}\left(\frac{x_{2}-s \cos \delta}{Y^{\prime}+s \sin \delta}\right)\right]\left.\right|_{0} ^{2} \\
& u_{3}^{\prime}=\frac{2 \alpha \mu b^{\prime}}{\pi}\left[-C s_{1} \sin \delta \ln T+D s_{2} \sin \delta \ln H+A s_{1}(d+s \sin \delta)\left(x_{2} \cos \delta-Y \sin \delta-s\right) \frac{1}{T^{2}}\right. \\
& +B s_{2}(d+s \sin \delta)\left(x_{2} \cos \delta-Y^{\prime} \sin \delta-s\right) \frac{1}{H^{2}} \\
& \left.-(A-C) s_{1} \cos \delta \tan ^{-1}\left(\frac{Y+s \sin \delta}{x_{2}-s \cos \delta}\right)-(B+D) s_{2} \cos \delta \tan ^{-1}\left(\frac{Y^{\prime}+s \sin \delta}{x_{2}-s \cos \delta}\right)\right]\left.\right|_{0} ^{2}
\end{aligned}
$$




\section{NUMERICAL RESULTS AND DISCUSSIONS}

We compare the displacement field due to a long vertical tensile fault of width $L$ its edge at the distance ${ }^{d}$ from the interface located in the isotropic half-space welded with orthotropic halfspace along the horizontal plane with the corresponding displacement field when both the halfspaces are isotropic. Therefore, we take $\delta=90^{\circ}$ (figure 1b). We assume the isotropic half-space to be poissonian so that $\sigma=0.25$. For the orthotropic

half-space, we use the values of elastic constants given by Love (1944). For Topaz,

$$
\begin{array}{lll}
c_{11}=2870, & c_{22}=3560, & c_{33}=3000, \\
c_{12}=1280, & c_{23}=900, & c_{13}=860, \\
c_{44}=1100, & c_{55}=1350, & c_{66}=1330,
\end{array}
$$

In terms of a unit of $10^{6}$ grammes $\mathrm{wt} / \mathrm{cm}^{2}$, this yields $a=1.2992$ and $b=0.8385$.

For Barytes,

$$
\begin{array}{llrl}
c_{11}=907, & c_{22}=800, & c_{33}=1074, \\
c_{12}=468, & c_{23}=273, & c_{13}=275, \\
c_{44}=122, & c_{55}=293, & c_{66}=283,
\end{array}
$$

In terms of a unit of $10^{6}$ grammes wt $/ \mathrm{cm}^{2}$, this yields $a=2.3118$ and $b=0.3735$.

When the lower half-space is also isotropic,

$$
\begin{aligned}
& c_{11}=c_{22}=c_{33}=\frac{2 \mu^{\prime}\left(1-\sigma^{\prime}\right)}{1-2 \sigma^{\prime}} \\
& c_{12}=c_{13}=c_{23}=\frac{2 \mu^{\prime} \sigma^{\prime}}{1-2 \sigma^{\prime}} \\
& c_{44}=c_{55}=c_{66}=\mu^{\prime}
\end{aligned}
$$

We take $\sigma^{\prime}=0.25 \quad c_{44} / \mu=$ and $\quad 0.5$ for numerical computations.

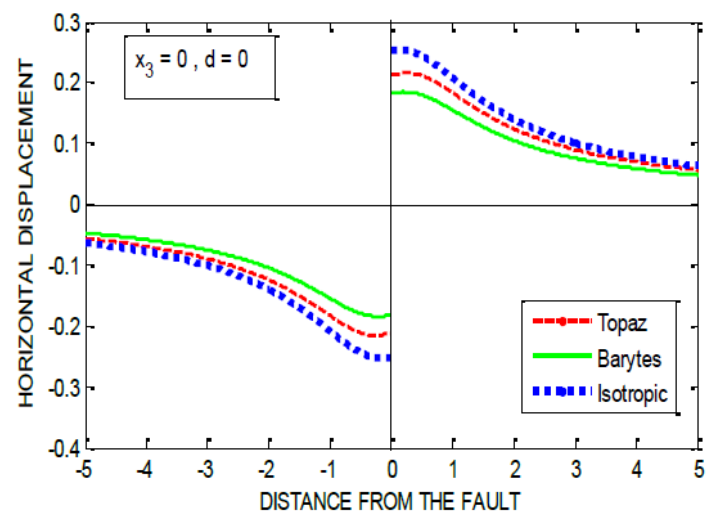

(a)

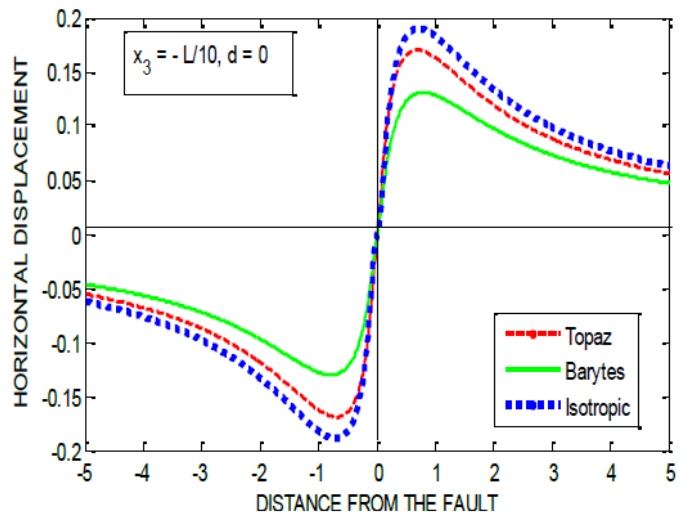

(b)

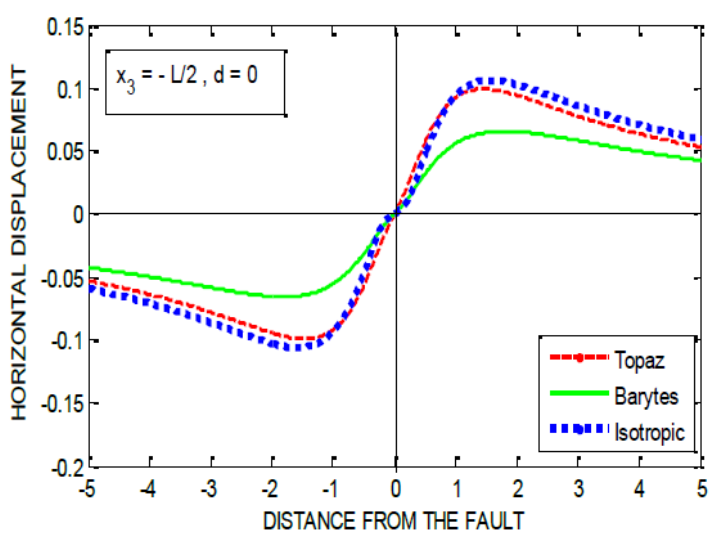

(c)

Figure 2 Variation of horizontal displacement $\left(u_{z}^{\prime} / b^{\prime}\right)$ with distance from the fault $\left(x_{z} / L\right)$ due to interface breaking tensile fault $(d=0)$ for (a) $x_{z}=0$, (b) $x_{z}=-L / 10$ and (c) $x_{z}=-L / 2$. 
Figure 2a-c display horizontal displacement $\left(u_{2}^{\prime} / b^{\prime}\right)$ with distance from the fault $\left(x_{2} / L\right)$ due to interface breaking tensile fault $(d=0)$. The horizontal displacement is anti-symmetric about the line $x_{2}=0$. In figure $2 \mathrm{a}$, the observer is at the interface, the horizontal displacement attains maximum value at origin. In figure $2 \mathrm{~b}-\mathrm{c}$, the observer is in the orthotropic half-space at $x_{3}=$ - L/10, $x_{3}=-L / 2$ respectively. The horizontal displacement for Barytes varies more significantly in magnitude rather than Topaz from the corresponding one for the isotropic case. It is observed that magnitude of horizontal displacement decreases when distance from the fault in orthotropic half-space increases.

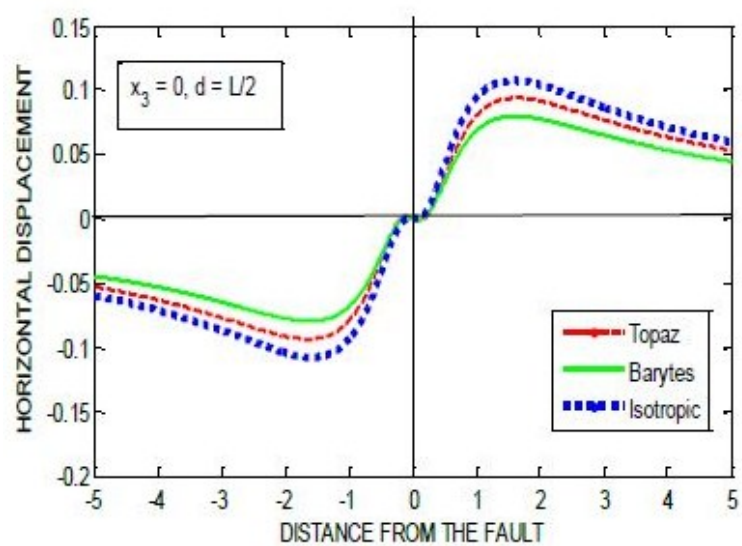

(a)

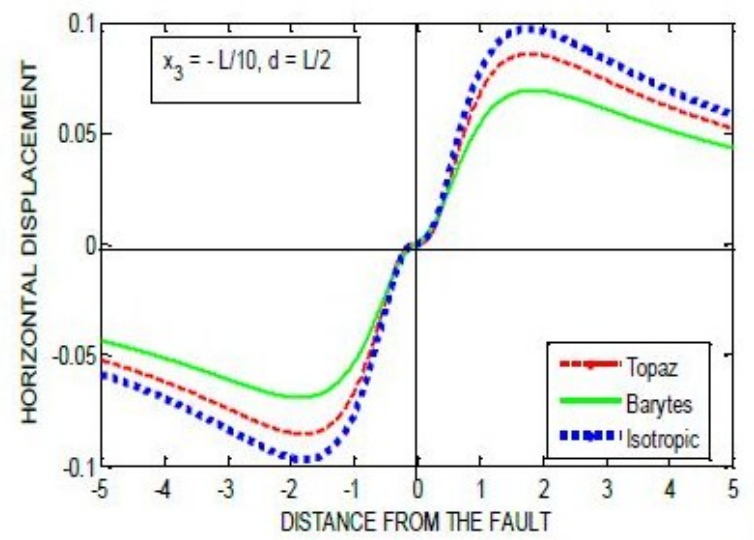

(b)

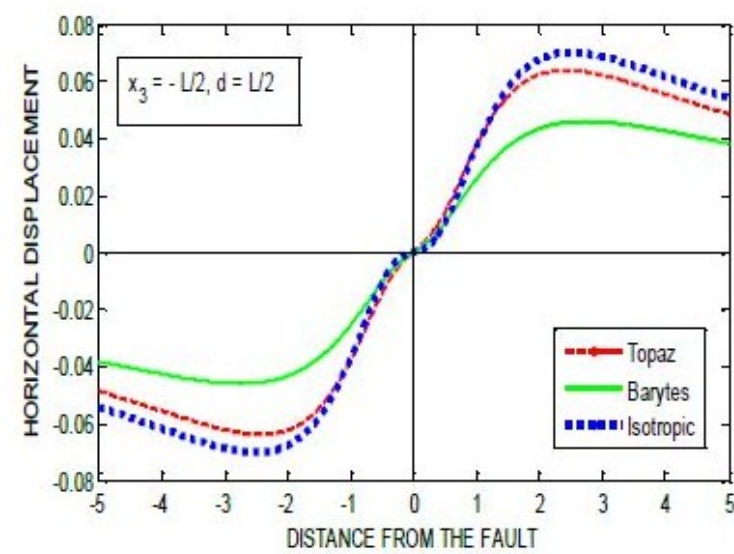

(c)

Figure 3a-c display horizontal displacement $\left(u_{z}^{\prime} / b^{\prime}\right)$ with distance from the fault $\left(x_{z} / L\right)$ located at a distance $d=L / 2$ from the interface for (a) $x_{z}=0$, (b) $x_{z}=-L / 10$ and (c) $x_{z}=-L / 2$.

In figure $3 a$, the observer is at the interface but In figure $3 b-c$, the observer is in the orthotropic half-space at $x_{3}=-L / 10, x_{3}=-L / 2$ respectively. We observe that magnitude of the displacement for Barytes is affected significantly, but the pattern is not affected much. It is also observed that the magnitude of horizontal displacement is maximum at the interface. 


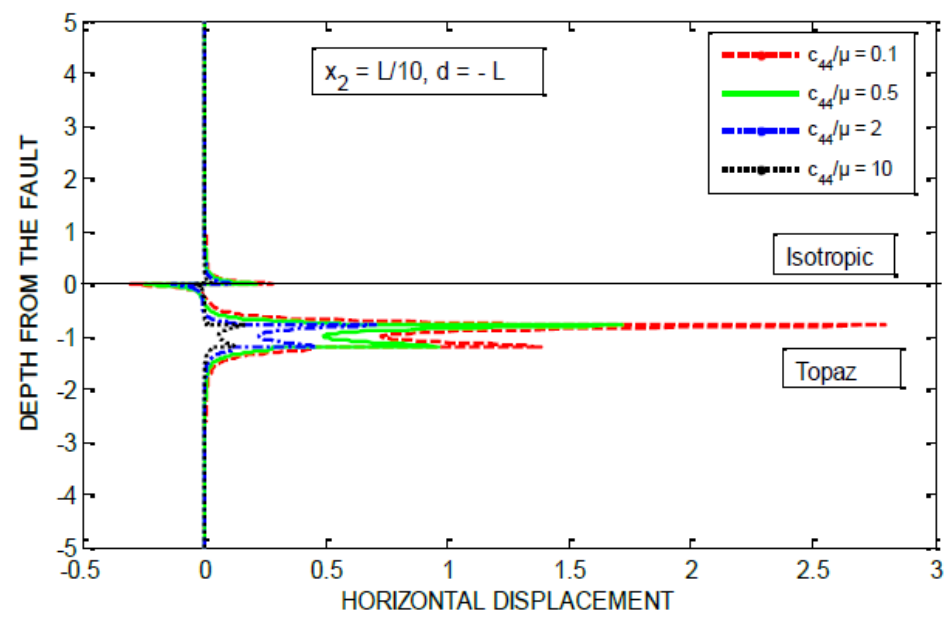

(a)

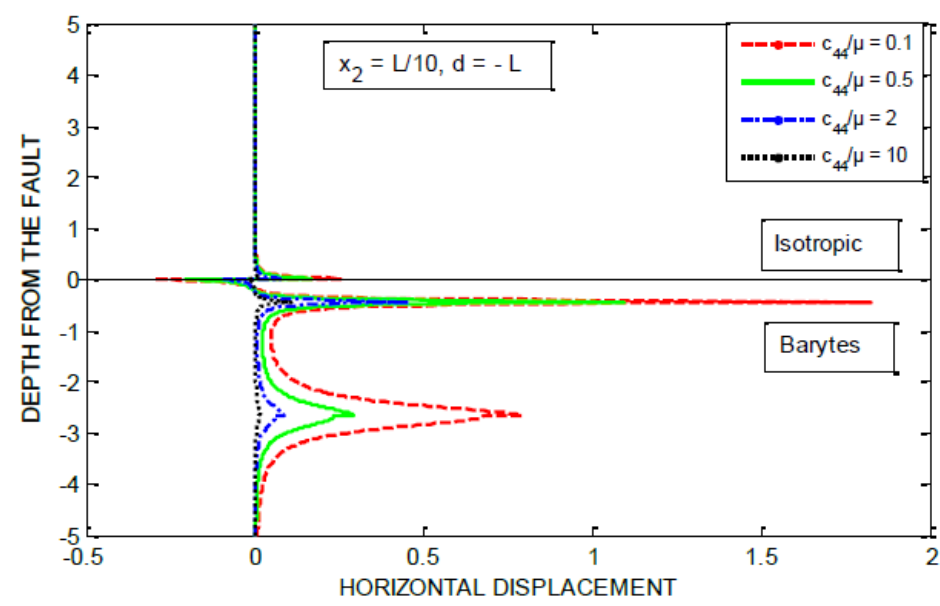

(b)

Figure 4 Variation of the horizontal displacement $\left(u_{z}^{\prime} / b^{\prime}\right)$ with depth from the fault $\left(x_{z} / L\right)$ at $x_{2}=L / 10$ at a distance $d=-$ L for different values of ratio of rigidities $\frac{s_{*}}{*}=0.1,0.5,2$ and 10 taking the orthotropic material as (a)Topaz, (b) Barytes. The magnitude of displacement is affected.

Figures $4 \mathrm{a}-\mathrm{c}$ show the variation of the horizontal displacement with depth $\left(x_{3} / L\right)$ for four values of rigidities $\frac{c_{44}}{\mu}=0.1,0.5,2$ and 10 for Topaz and Barytes respectively due to tensile fault located at a distance $d=-L$ from the interface. For all values of ratio of rigidities, the horizontal displacement attains maximum value at the lower end of the fault and minimum value at the upper end of the fault. It is observed that with increase in the value of ratio of rigidities, there is decrease in horizontal displacement. Also, for the same value of ratio of rigidities horizontal displacement for Topaz is more than barytes in magnitude.
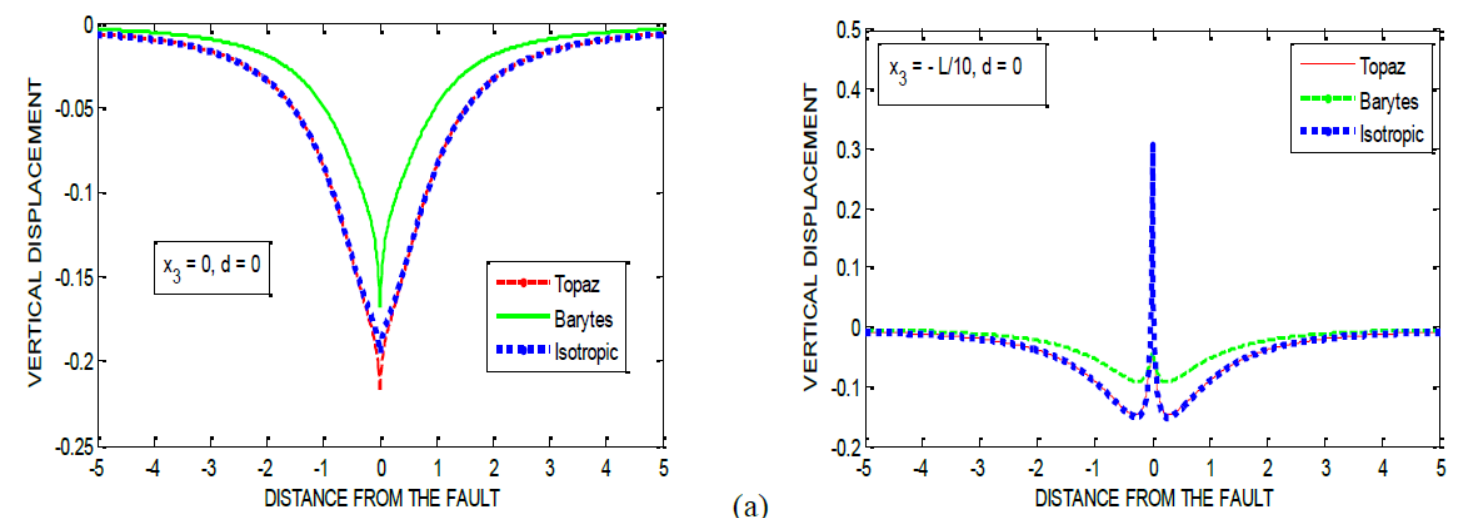

(a) 


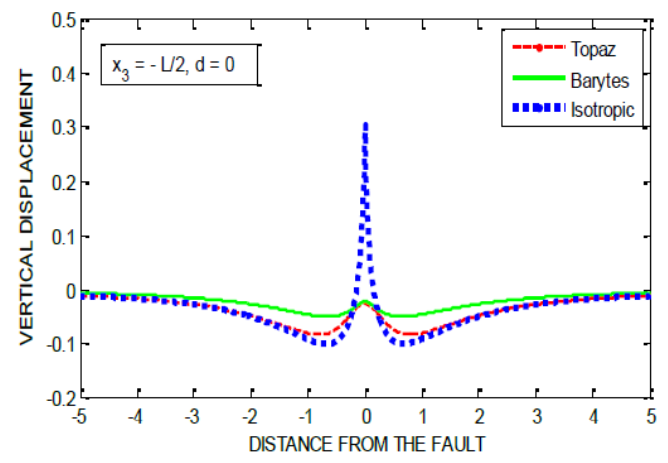

(c)

Figure 5 Variation of vertical displacement $\left(u_{z}^{\prime} / b^{\prime}\right)$ with distance from the fault $\left(x_{z} / L\right)$ due to interface breaking tensile fault (d=0) for (a) $x_{z}=0$, (b) $x_{z}=-L / 10$ and (c) $x_{z}=-L / 2$.

Figure 5a-c display vertical displacement $\left(u_{3}^{\prime} / b^{\prime}\right)$ with distance from the fault $\left(x_{2} / L\right)$ due to interface breaking tensile fault $(d=0)$. The vertical displacement is symmetric about the line $x_{2}=0$. In figure $5 \mathrm{a}$, the observer is at the interface, the vertical displacement attains minimum value at origin. In figure $5 \mathrm{~b}-\mathrm{c}$, the observer is in the orthotropic half-space at $x_{3}=-L / 10, x_{3}=-L / 2$ respectively. The vertical displacement for Barytes varies more significantly in magnitude rather than Topaz from the corresponding one for the isotropic case.

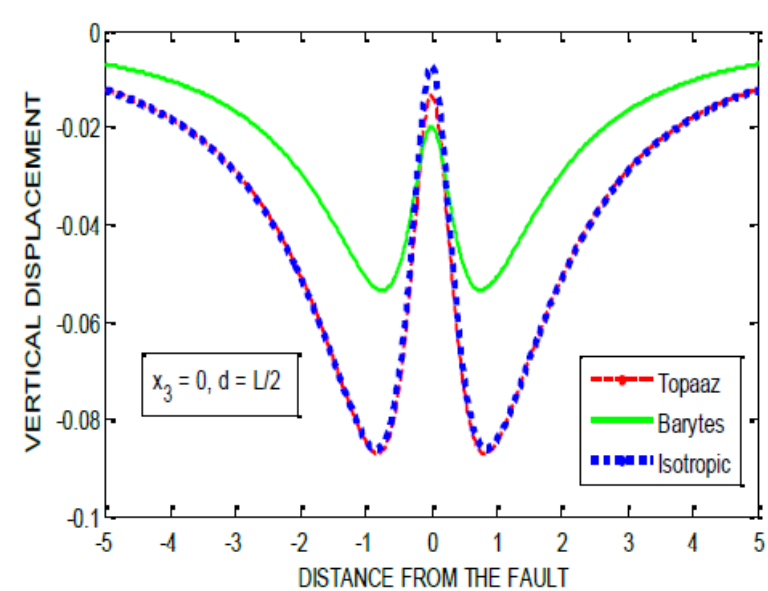

(a)

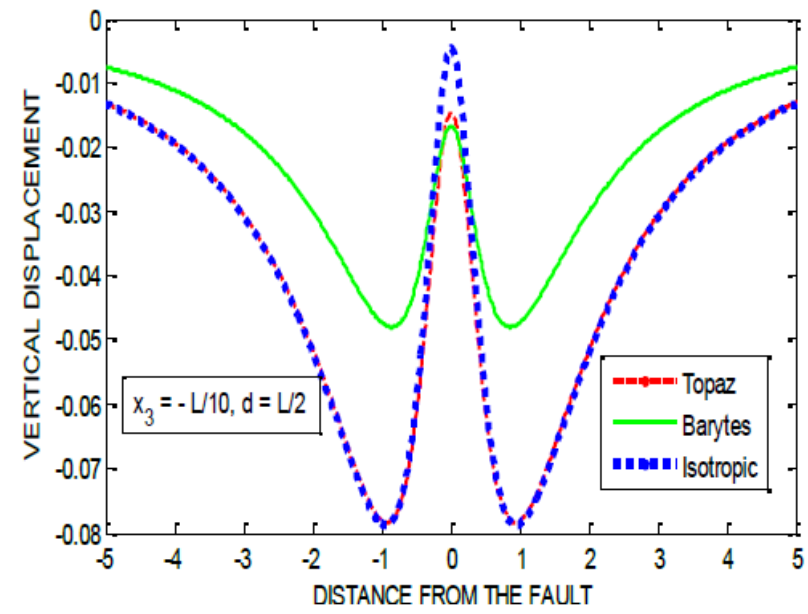

(b)

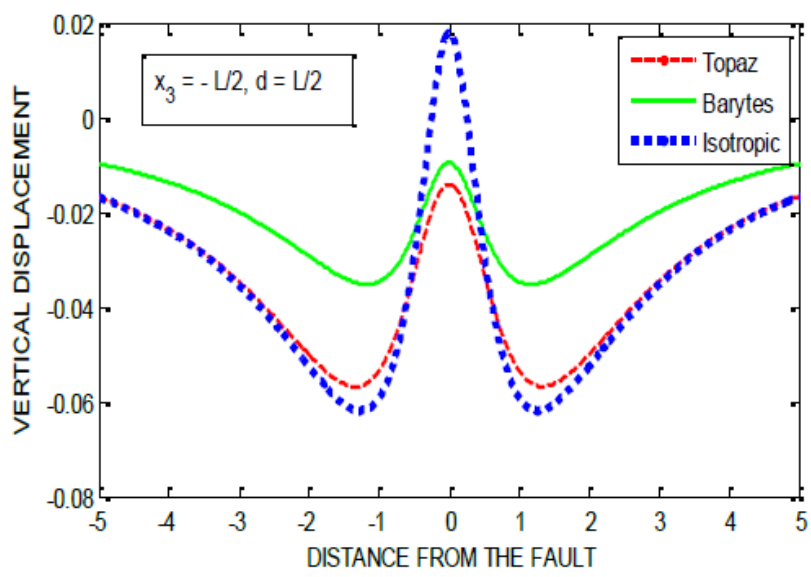

(c)

Figure 6 Variation of vertical displacement $\left(u_{z}^{\prime} / b^{\prime}\right)$ with distance from the fault $\left(x_{z} / L\right)$ due to a long vertical tensile fault at a distance $d=L / 2$, for (a) $x_{z}=0$, (b) $x_{z}=-L / 10$ and (c) $x_{z}=-L / 2$. 
Figure 6a-c display vertical displacement $\left(u_{3}^{\prime} / b^{\prime}\right)$ with distance from the fault $\left(x_{2} / L\right)$ located at a distance $d=L / 2$ from the interface. In figure $6 \mathrm{a}$, the observer is at the interface but In figure $2 \mathrm{~b}$ c, the observer is in the orthotropic half-space at ${ }^{x_{3}}=-L / 10,{ }^{x_{3}}=-L / 2$ respectively. We observe that magnitude of the displacemet for Barytes is affected significantly, but the pattern is not affected much. It is also observed that the magnitude of vertical displacement is maximum at the interface.

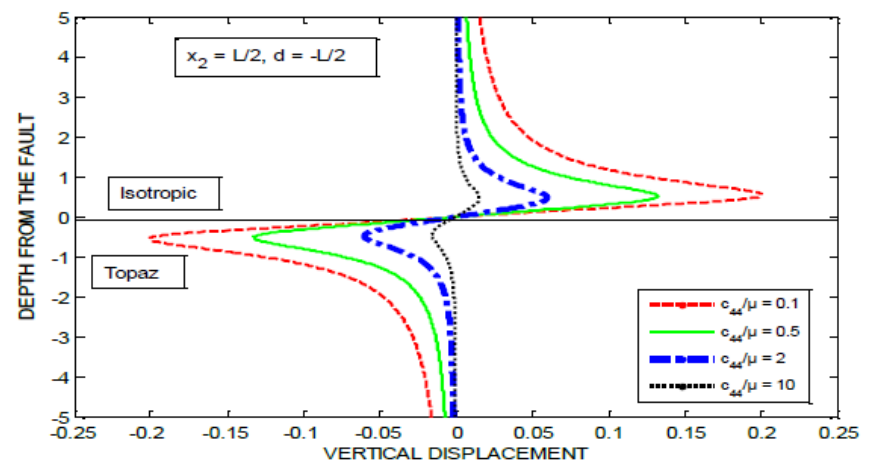

(a)

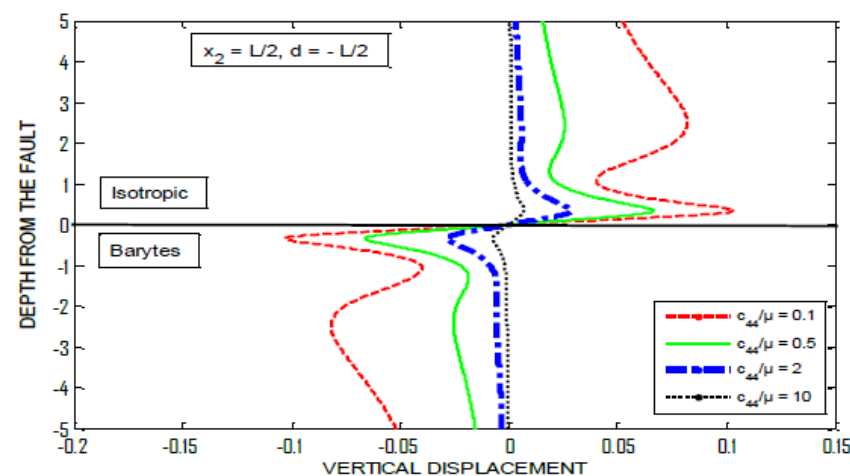

(b)

Figure 7 Variation of the vertical displacement $\left(u_{z}^{\prime} / b^{\prime}\right)$ with depth from the fault $\left(x_{z} / L\right)$ at $x_{2}=L / 2$ at a distance $d=-L / 2$ for different values of ratio of rigidities $\frac{s_{\mu}}{\mu}=0.1,0.5,2$ and 10 taking the orthotrpic material as (a) Topaz (b) Barytes. The magnitude of displacement is affected.

Figures $7 \mathrm{a}-\mathrm{c}$ show the variation of the vertical displacement with depth $\left(x_{3} / L\right)$ for four values of rigidities $\frac{c_{44}}{\mu}=0.1,0.5,2$ and 10 for Topaz and Barytes respectively due to tensile fault located at a distance $\mathrm{d}=-L / 2$ trom the interface. For all values of ratio of rigidities, the vertical displacement attains maximum value at the lower end of the fault and minimum value at the upper end of the fault. It is observed that with increase in the value of ratio of rigidities, there is decrease in vertical displacement. Also, for the same value of ratio of rigidities vertical displacement for Topaz is more than barytes in magnitude.

Now, we also considering surface plots for horizontal and vertical displacement of surface due to a long vertical tensile fault of width $L$.
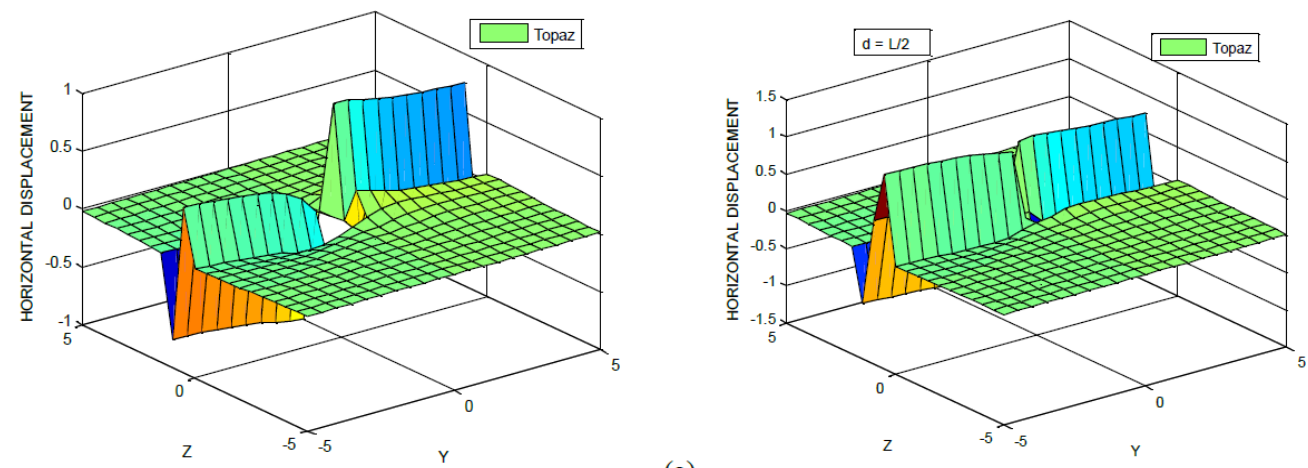

(a)

Figure 8 Horizontal displacement of the surface for Topaz due to vertical tensile fault located at (a) interface breaking fault (b) $d=L / 2$. 

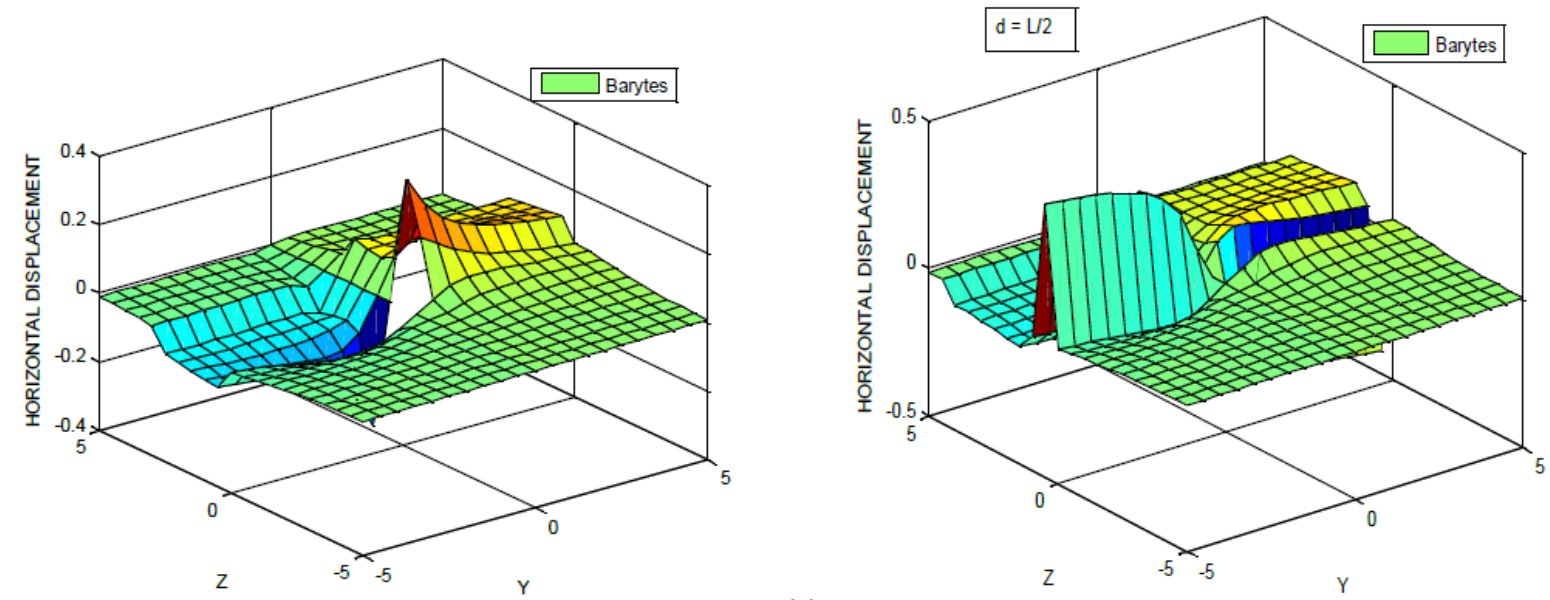

(a)

(b)

Figure 9 Horizontal displacement of the surface for Barytes due to vertical tensile fault located at (a) interface breaking fault (b) $d=L / 2$.
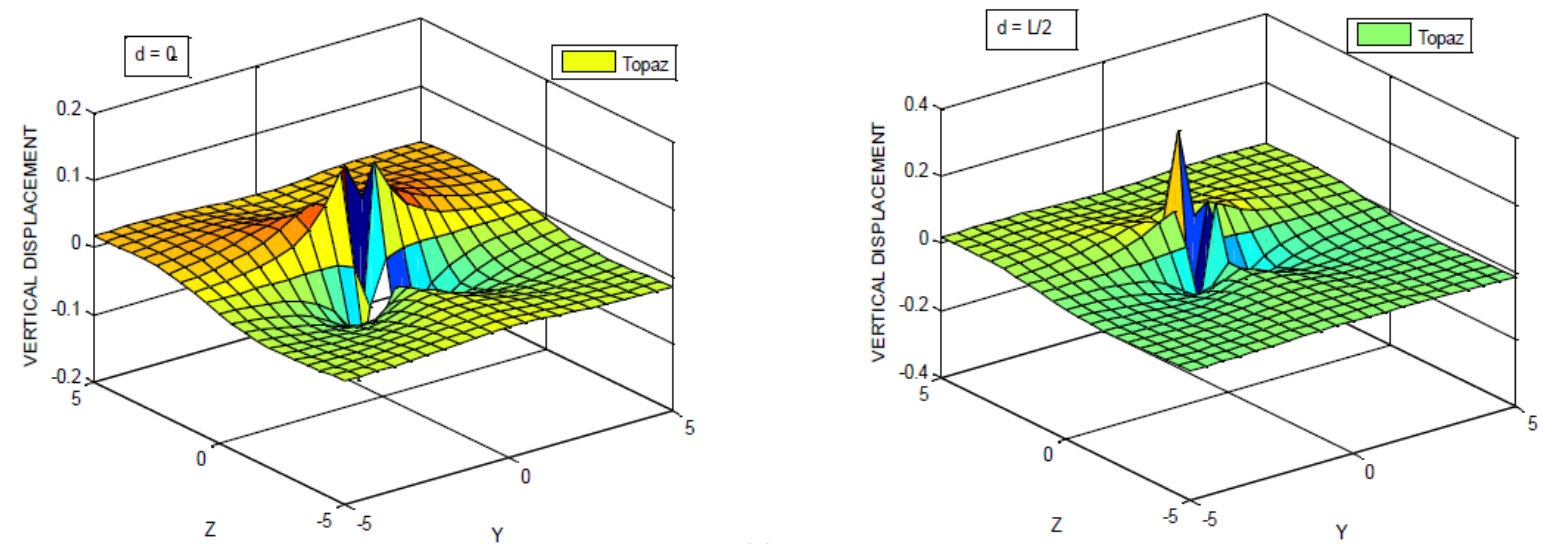

(a)

Figure 10 Horizontal displacement of the surface for Isotropic half-space due to vertical tensile fault located at (a) interface breaking fault (b) $\mathrm{d}=L / 2$
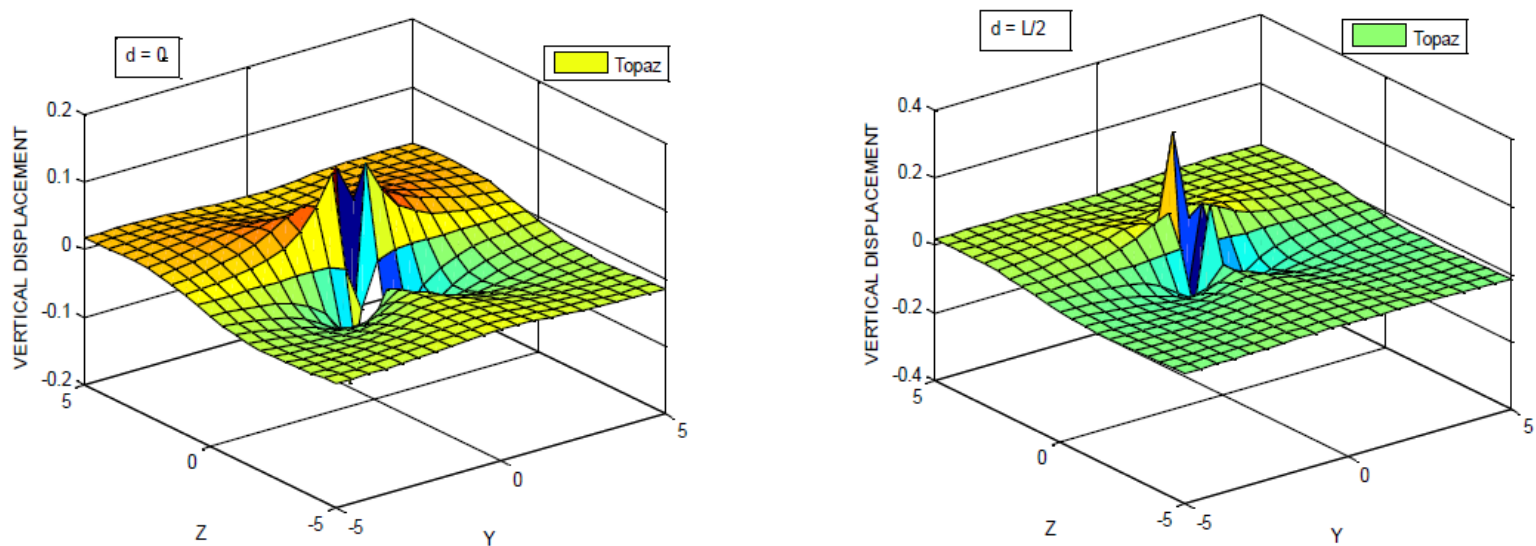

(a)

Figure 11 Vertiacal displacement of the surface for Topaz due to vertical tensile fault located at (a) interface breaking fault (b) $d=L / 2$. 


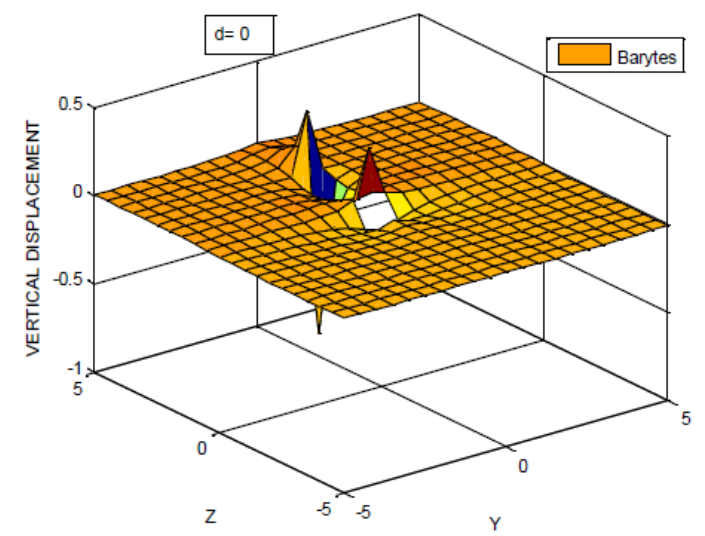

(a)

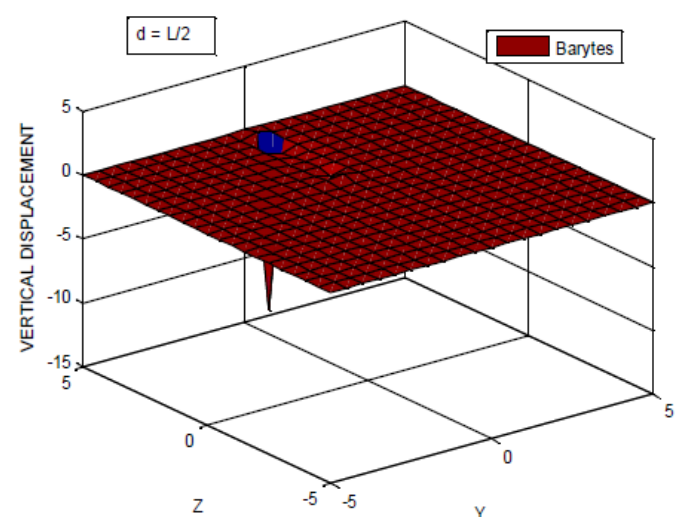

(b)

Figure 12 Horizontal displacement of the surface for Topaz due to vertical tensile fault located at (a) interface breaking fault (b) $d=L / 2$.
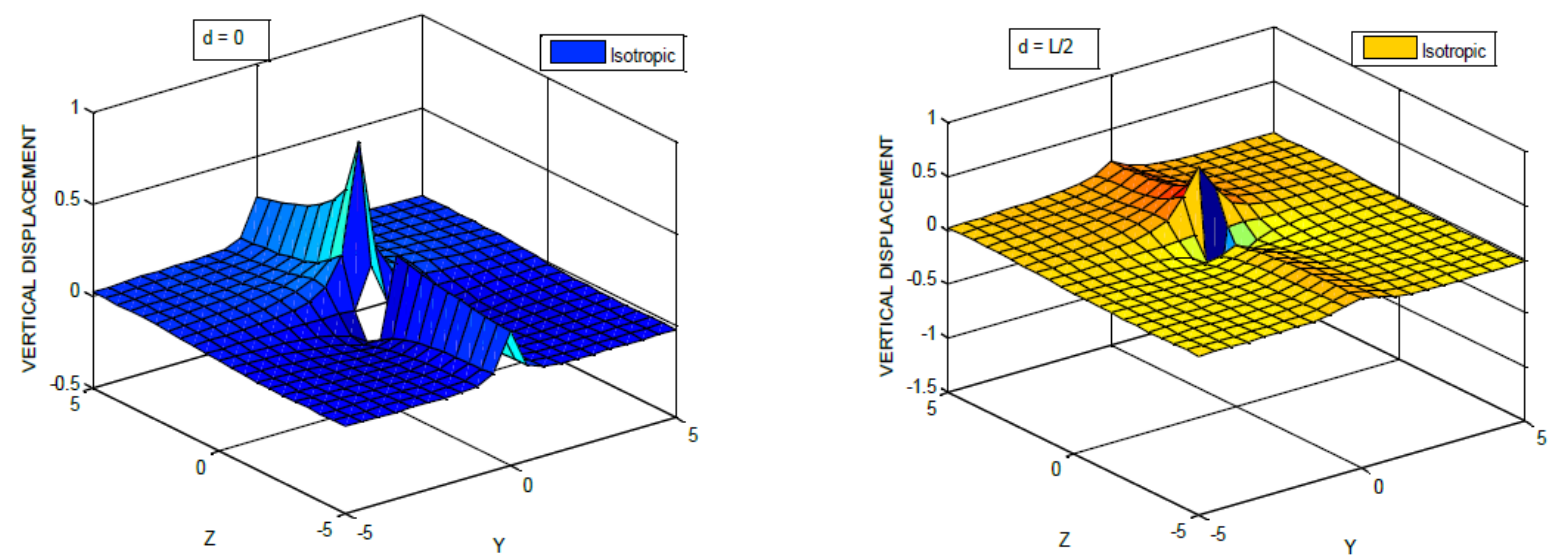

(a)

Figure 13 Horizontal displacement of the surface for Topaz due to vertical tensile fault located at (a) interface breaking fault (b) $d=L / 2$.

From all figures, we observe that horizontal and vertcal displacement of the surface at the interface breaking fault is more pronounced than when tensile fault is at depth $d=L / 2$.

\section{CONCLUSION}

We have obtained the stresses and displacements for a long tensile fault of finite width L located in the isotropic half-space overlying the orthotropic half-space. The results obtained here satisfy the necessary continuity conditions

$$
\begin{array}{ll}
p_{23}=p_{23}^{\prime}, & p_{33}=p_{33}^{\prime} \\
u_{2}=u_{2}^{\prime}, & u_{3}=u_{3}^{\prime}
\end{array}
$$

at $x_{3}=0$ for the two half-spaces to be in welded contact along the plane $x_{3}=0$. Moreover, when the orthotropic half-space is replaced by isotropic one, the results of the present paper, in the limit, coincide with the corresponding results of Kumar et al. (2005) for two half-spaces to be in welded contact. Numerical results presented the variation of horizontal displacement, vertical displacement with distance and depth fom the fault for different distances of fault from the interface. Also displacements of the surface presented graphically. Numerical computations indicate that the deformation field due to a source in an isotropic half- space in welded contact with an anisotropic half-space may differ substantially from the deformation field when both the half-spaces are isotropic. 


\section{ACKNOWLEDGEMENT}

I, Yogita Godara (JRF), am grateful to the council of scientific and Industrial Research, New Delhi for financial support. The authors are thankful to the referees for their comments which led to an improvement in the presentation of the paper.

\section{REFERENCES}

[1] Steketee, J.A, On Volterra's Dislocations in a Semi-infinite Elastic Medium, Can. J. Phys., vol. 36,1958a, 192-205.

[2] Stekettee, J.A, Some Geophysical Applications of the Elasticity Theory of Dislocations, Can. J. Phys., vol. 36,1958b, 1168-1198.

[3] Chinnery, M. A., The Deformation of the Ground Around Surface Faults, Bull. Seismol. Soc. Am., vol. 61, 1961, 355-372.

[4] Sipkin, S. A,. Interpretation of non-double- couple earthquake mechanisms derived from moment tensor inversions, J. Geophys. Res., 91, 531-547.

[5] Maruyama, T., Statical elastic dislocations in an infinite and semi-infinite medium, Bull. Earthquake Res. Inst., 42, 1964, 289-368.

[6] Freund, L. B. and D. M. Barnett, A two-dimensional analysis of surface deformation due to dip-slip faulting, Bull. Seismol. Soc. Am., 66, 667-675, 1976.

[7] Davis, P. M., Surface deformation associated with a dipping hydrofracture, Geophys. J. Res., 88, 1983, 5826-5836.

[8] Singh, S. J. \& Garg, N. R., 1986 On the representation of two-dimensional seismic sources, Acta Geophy. Pol., 34, 1-12.

[9] Rani, S., S. J. Singh, and N. R. Garg," Displacements and stresses at any point of a uniform half-space due to two-dimensional buried sources", Phys. Earth Planet. Inter. 65, 276-286, 1991. http://dx.doi.org/10.1016/0031-9201 (91) 90134-4.

[10] $\mathrm{Wu}$, R.S. \& Chou, Y.T., Line force in a two-phase orthotropic medium, ASME. Appl. Mech., 49, 1982, 55-61.

[11] Singh, S. J., Static deformation of a transversely isotropic multilayered half-space by general surface loads, Phys. Earth Planet. Inter., 42, 1986, 263-273.

[12] 12. Pan, E., Static response of a transversely isotropic and layered half-space due to general surface loads, Phys. Earth Planet. Inter., 54, 1989a, 353-363.

[13] 13. Pan, E., Static response of a transversely isotropic and layered half-space due to general surface loads, Phys. Earth Planet. Inter., 58, 1989b, 103-117.

[14] Garg, N. R., S. J. Singh, and S. Manchanda," Static deformation of an orthotropic multilayered elastic half-space by two dimensional surface loads", Proc. Ind. Acad. Sci. (Earth Planet. Sci.), 100, 205-218, 1991.

[15] Singh, S. J., S. Rani, and N. R. Garg," Displacements and stresses in two welded halfspaces due to two- dimensional sources", Phys. Earth. Planet. Inter 1991.

[16] Kumar, A., Singh, S. J. \& Singh, J., Deformation of two welded half-spaces due to a long inclined tensile fault, J. Earth Syst. Sci., 114, 2005, 97-103.

[17] Singh, S. J. \& Rani, S., Static deformation due to two-dimensional seismic sources embedded in an isotropic half-space in welded contact with an orthotropic half space, $J$. Phys. Earth, 39, 1991, 599- 618. 18. Singh, M. \& Singh, S. J., Static deformation of a uniform half-space due to a very long tensile fault, ISET J. Earthquake Techn. 37, 2000, 27 38. 